\title{
Conventional Treatment of Hypoparathyroidism
}

\author{
Muriel Babey, MD ${ }^{a}$, Maria-Luisa Brandi, MD, PhD ${ }^{b}$, Dolores Shoback, MD ${ }^{c}$ *
}

\section{KEYWORDS}

- Hypoparathyroidism • Hypocalcemia • Serum calcium • PTH • Calcitriol

- Serum phosphate

\section{KEY POINTS}

- Conventional therapy of hypoparathyroidism consists of oral calcium and activated vitamin $\mathrm{D}$ or vitamin $\mathrm{D}$ supplements at doses adjusted to meet the needs of the patient.

- Therapeutic goals are to prevent symptoms and signs of hypocalcemia; to maintain low normal calcium and normal phosphate levels and a calcium-phosphate product less than $55 \mathrm{mg}^{2} / \mathrm{dL}^{2}$; and to avoid hypercalciuria, hypercalcemia, and end-organ complications such as renal and other extraskeletal calcifications.

- Calcium carbonate is better absorbed at a low gastric $\mathrm{pH}$ level achieved at meal times. Calcium citrate is a better choice for patients taking proton pump inhibitors or $\mathrm{H} 2$ agonists and can be taken with or without meals.

- Calcitriol or alfacalcidol, both activated forms of vitamin D, are considered the treatments of choice for most patients with hypoparathyroidism.

- Diets rich in calcium may reduce the amount of calcium supplements needed, but phosphate intake needs to be considered carefully.

\section{INTRODUCTION}

Hypoparathyroidism leads to hypocalcemia, hyperphosphatemia, and elevated fractional excretion of calcium in the urine, due to either absent or inadequately low circulating concentrations of parathyroid hormone (PTH). Conventional therapy of hypoparathyroidism consists of oral calcium and activated vitamin $D$ or vitamin $D$

Disclosure Statement: Dr D. Shoback has received consulting fees from Shire Pharmaceuticals and Ascendis Pharmaceuticals. Dr M.L. Brandi has received consulting fees and grant support from Alexion, Abiogen, Amgen, Eli Lilly, and Shire Pharmaceuticals. Dr M. Babey has nothing to disclose.

a Department of Medicine, Highland Hospital, 1411 East 31st Street, Oakland, CA 94602, USA;

b Department of Surgery and Translational Medicine, University of Florence, Viale Pieraccini 6, 50139 Florence, Italy; ' Endocrine Research Unit, Department of Medicine, San Francisco Department of Veterans Affairs Medical Center, University of California, San Francisco, $111 \mathrm{~N}$, 1700 Owens Street, 3rd Floor Room 369, San Francisco, CA 94158, USA

* Corresponding author.

E-mail address: Dolores.shoback@ucsf.edu 